\title{
Effects of Knee Malalignment on Static and Dynamic Postural Stability
}

\author{
Yun-Won Chae', Ji-Won Park², Seol Park² \\ 'Department of Physical Therapy, Gwangju Health College, Gwangju; ${ }^{2}$ Department of Pphysical Therapy, College of Medical Health, Catholic \\ University of Daegu, Gyeongsan, Korea
}

Purpose: This study was conducted in order to determine the effects of knee malalignment including genu varum, valgum, and recurvatum on static and dynamic postural stability.

Methods: A total of 80 subjects were enrolled in this study. Subjects who showed over $3 \mathrm{~cm}$ in the distance between the knees were classified as the genu varum group, and subjects who showed over $3 \mathrm{~cm}$ in the distance between the ankles were classified as the genu valgum group. Subjects who showed over $1 \mathrm{~cm}$ in the distance between the patella and a table in prone position were classified as the genu recurvatum group. Static and dynamic stability were measured as overall, anterioposterior, and mediolateral balance index using a Biodex Balance System.

Results: This study showed that knee alignment affected static and dynamic postural stability. In particular, there were significant differences in the mediolateral stability index among genu varum, valgum group, and the other groups, but no differences in overall and anteriolateral stability index. Significant differences in the anterioposterior stability index were observed between genu recurvatum and the other groups, however, there were no differences in overall and mediolateral stability index.

Conclusion: The findings were that knee malalignment affects postural stability toward a specific direction. Treatment to improve postural stability for treatment of knee malalignment or to prevent falling or injuries is needed and postural stability toward a specific direction according to the knee alignment conditions should be considered.

Keywords: Postural balance, Bone malalignment, Genu varum, Genu valgum

\section{서 론}

낙상은 자세 조절 시스템이 평형을 유지하는 데 실패했을 때 일어난 다. 불량한 하지 정렬과 같은 근골격계의 변화는 이러한 자세 조절 능력을 저하시키고 평형을 유지하는 데에 어려움을 증가시켜 낙상을 유발한다.2-6 엉덩관절-무릎관절-발목관절에 이르는 하지의 역학적 축의 정렬은 중력에 대항해 신체를 지지하는 데 요구되며, 이는 신체 균형을 조절하기 위한 자세적 조절의 필수 요소이다.-9 따라서 하지 정렬은 자세적 안정성을 향상시키기 위해 고려되어야 하며, 낙상이나 부상을 예측하고 예방하기 위해 중요하다.10-12

하지의 역학적 축이나 체중 지지선은 무릎관절의 중심을 통과하 며, 무릎의 하중 분포에 영향을 미친다.13-14 따라서 하지 정렬이 불량
할 경우 하지의 역학적 축이 이동하게 되고, 이는 자세적 동요를 증가 시켜 불안정성을 야기시킨다.15-17 특히, 안굽이 무릎(genu varum)은 무릎의 안쪽 구획에 하중을 증가시키고 안쪽 관절 간격이 소실되는 반면, 밖굽이 무릎(genu valgum)은 무릎의 가쪽 구획에 하중을 증가 시키고 가쪽 관절 간격이 소실된다.18 하중분포에 대한 하지 정렬의 이러한 기계적 효과는 뼈관절염(osteoarthritis)을 증가시키고 신체 기 능을 감소시킨다. 무릎 변형, 특히 안굽이 무릎이 일반적으로 낙상 문 제를 가지는 노인들에게서 흔히 발견된다는 것을 고려할 때 무릎관 절 정렬과 자세적 안정성과의 관계는 중요하다.17

Samaei 등 ${ }^{15}$ 은 안굽이 무릎이 정적, 동적인 상황 하에서 내외측 안 정성(mediolateral stability)에 영향을 미친다고 하였으나, Chae 등 ${ }^{19}$ 은 동적인 상황에서는 내외측 안정성 뿐만 아니라 전후측 안정성(ante-
Received January 8, 2015 Received February 2, 2015

Accepted February 9, 2015

Corresponding author Seol Park

E-mail parksul85@hanmail.net

- The Research has been conducted by the Research Grant of Gwangju Health College in 2013(No. 3013010).
Copylight @2015 The Korean Society of Physical Therapy

This is an Open Access article distribute under the terms of the Creative Commons Attribution Non-commercial License (Http:// creativecommons.org/license/by-nc/3.0.) which permits unrestricted non-commercial use, distribution, and reproduction in any medium, provided the original work is properly cited. 
rioposterior stability)과 전체 안정성(overall stability)에도 영향을 미친 다고 하였다. Siqueira 등 10 은 과다폄된 무릎의 경우 바로 선 자세에서 의 압력중심(Center of pressure, COP) 이동 속도가 높은 것으로 보아, 과다폄(hyperextension)된 무릎이 자세적 조절이나 무릎의 안정성에 영향을 미친다고 하였다. 선행 연구에서는 안굽이 무릎이 내외측 안 정성에 영향을 미쳤다고 하였으므로, 무릎 정렬의 편차가 특정한 방 향으로의 안정성에 영향을 미칠 수 있음을 시사하였다. 따라서 전두 면(frontal plane)에서의 부정렬로 나타나는 안굽이 무릎 이외에도 밖 굽이 무릎이 자세적 안정성에 미치는 영향을 알아볼 필요가 있으며, 시상면(sagittal plane)에서의 부정렬로 나타나는 젖힌 무릎(genu recurvatum)에 대해서도 연구해 볼 필요가 있다. 본 연구에서는 선행 연 구에서 일치되지 않은 실험 조건을 통제하고 그에 따른 연구 결과를 명확히 알아봄으로써 무릎 정렬 편차와 정적 및 동적 안정성과의 관 계를 규명하고자 하였다. 이를 위해 대표적인 무릎 정렬 이상인 안굽 이 무릎, 밖굽이 무릎, 그리고 젖힌 무릎이 정적 및 동적인 상황 하에 서 자세 안정성에 어떠한 영향을 미치는지 알아보고자 하였다.

\section{연구방법}

\section{1. 연구대상}

본 연구에서는 무릎 정렬 편차에 따른 정적, 동적 자세 안정성을 알아 보기 위해 대상자들을 각 20 명의 여자로 구성된 네 그룹(안굽이 무 릎군, 밖굽이 무릎군, 젖힌 무릎군, 그리고 대조군)으로 분류하였다 (Table 1). 안굽이 무릎과 밖굽이 무릎을 가진 대상자를 선별하기 위 해 맨발의 상태로 해부학적 자세를 취하여 벽을 등지고 선 후 무릎과 발목 간의 거리를 측정하였다. 우선 등과 둔부, 그리고 발의 뒤꿈치를 벽에 대고 바로 선 상태로 발을 서로 모으라고 주문했을 때, 발목의 안쪽 복사뼈 사이가 $3 \mathrm{~cm}$ 이상이면 밖굽이 무릎으로 규정하고, 무릎 의 안쪽 융기 사이가 $3 \mathrm{~cm}$ 이상이면 안굽이 무릎으로 규정하였다. ${ }^{15}$ 젖힌 무릎을 가진 대상자를 선별하기 위해 대상자를 테이블 위에 엎 드려 눕게 한 후 무릎과 지면 사이의 간격을 측정하였다. 우선 발을
테이블 밖으로 위치하도록 엎드려 누웠을 때 무릎이 지면에 맞닿아 있지 않거나 $1 \mathrm{~cm}$ 이상일 경우를 젖힌 무릎으로 판단하였다.

대상자들의 일반적인 특성을 알아보기 위해 신장계(ZENIX, Korea)를 이용하여 키와 몸무게를 측정하였으며, 실험 전 대상자에게 실 험에 대해 충분히 설명한 후, 대상자들의 자발적 동의를 얻어 진행하 였다.

\section{2. 실험방법}

1) 측정 방법

자세 안정성을 측정하기 위해 Biodex Balance System (Biodex Inc., USA)을 사용하여 전체균형지수(Overall Stability Index, OSI), 전후균 형지수(Anterioposterior Stability Index, APSI), 내외측 균형지수(Mediolateral Stability Index, MLSI)를 측정하였다. 정적인 자세안정성을 측정하기 위해 발판이 고정되어 있는 상태에서 모니터에 나온 동심 원의 중심에 스스로 자신의 $\mathrm{COP}$ 를 위치시키도록 한 후 검사를 시작 하였다. 20 초 동안 2 회 측정하였으며, 중간에 10 초의 휴식을 가졌다. 반면, 동적 안정성은 고정되어 있지 않은 발판 위에서 측정하였다. 발 판의 불안정성은 1-12단계까지로 다양하게 조절할 수 있도록 되어 있 으며, 12 단계가 가장 안정적이고 1단계가 가장 불안하다. 본 실험에서 는 5 단계를 이용해 실시하였다. 발판이 5 단계로 설정된 상태에서 모 니터에 나온 동심원의 중심에 스스로 자신의 $\mathrm{COP}$ 를 위치시킨 후 검 사를 시작하였다. 20 초 동안 2 회 측정하였으며 중간에 10 초의 휴식을 가졌다. 측정값은 OSI, APSI, MLSI로 기록하였다.

\section{2) 통계 처리}

네 그룹의 일반적인 특성을 알아보기 위해 기술적 통계를 사용하였 으며, 네 그룹 사이에 무릎 간 간격, 발목 간 간격, 무릎과 지면과의 간 격, 그리고 정적 및 동적인 상태 하에서의 OSI, APSI, MLSI의 차이를 알아보기 위해 one-way ANOVA를 사용하여 분석하였다. 사후분석 은 Tuckey를 사용하였으며, 유의수준(a)은 0.05 로 하였다.

Table 1. Characteristics of the subjects (Mean \pm SD)

\begin{tabular}{|c|c|c|c|c|}
\hline & Control & Genu varum & Genu valgum & Genu recurvatum \\
\hline $\mathrm{N}$ & 20 & 20 & 20 & 20 \\
\hline Age (year) & $21.0 \pm 0.9$ & $21.3 \pm 1.0$ & $21.6 \pm 1.3$ & $21.3 \pm 1.2$ \\
\hline Height (cm) & $163.5 \pm 3.3$ & $162.5 \pm 4.7$ & $161.6 \pm 4.8$ & $163.6 \pm 4.3$ \\
\hline Weight $(\mathrm{kg})$ & $54.6 \pm 4.3$ & $54.3 \pm 6.0$ & $53.0 \pm 4.5$ & $55.9 \pm 4.9$ \\
\hline Distance between knees $(\mathrm{cm})^{*}$ & $1.0 \pm 1.0$ & $8.3 \pm 1.1$ & $0.0 \pm 0.0$ & $1.0 \pm 1.0$ \\
\hline Distance between ankles $(\mathrm{cm})^{*}$ & $1.4 \pm 1.0$ & $0.0 \pm 0.0$ & $9.6 \pm 1.1$ & $1.1 \pm 1.0$ \\
\hline Distance between a patella and a table $(\mathrm{cm})^{*}$ & $0.0 \pm 0.0$ & $0.0 \pm 0.0$ & $0.0 \pm 0.0$ & $2.0 \pm 0.5$ \\
\hline
\end{tabular}

Values are presented as mean \pm standard deviation. ${ }^{*} \mathrm{p}<0.01$. 
결 과

\section{1. 무릎 정렬 편차가 정적 안정성에 미치는 영향}

정적인 자세 안정성을 측정한 결과 네 그룹 사이에 OSI에서는 유의한 차이가 없었다.

APSI를 알아본 결과 젖힌 무릎군과 대조군 사이에 유의한 차이를 보여 젖힌 무릎군의 전후 안정성이 유의하게 떨어진 것으로 나타났 다. 안굽이 무릎군, 밖굽이 무릎군 또한 대조군에 비해 전후 안정성 이 떨어진 것으로 보이나, 유의한 차이는 없었다.

MLSI를 알아본 결과 안굽이 무릎군과 밖굽이 무릎군에서 다른 두 그룹에 비해 유 의한 차이를 보였다. 안굽이 무릎과 밖굽이 무릎 은 대표적인 전두면에서의 무릎 정렬 이상으로, 내외측 안정성에 유 의한 영향을 미치는 것으로 나타났다(Table 2, Figure 1A).

\section{2. 무릎 정렬 편차가 동적 안정성에 미치는 영향}

동적인 자세 안정성을 측정한 결과 네 그룹 사이에 OSI에서는 유의한 차이가 없었으며, 이는 정적 안정성에서 나타난 결과와 일치하였다.

$\mathrm{APSI}$ 를 알아본 결과 젖힌 무릎군이 나머지 세 그룹과 유의한 차이 를 보였으며, 이는 젖힌 무릎이 전후 안정성에 유의한 영향을 미치는
것으로 나타났다.

MLSI를 알아본 결과 안굽이 무릎군과 밖굽이 무릎군에서 다른 두 그룹에 비해 유의한 차이를 보였으며, 이는 정적 안정성에서 나타 난 결과와 일치하였다(Table 2, Figure 1B).

\section{고 찰}

본 연구는 20 명의 여자로 구성된 네 그룹, 즉, 안굽이 무릎군, 밖굽이 무릎군, 젖힌 무릎군, 그리고 대조군 사이에 정적 및 동적인 자세 안 정성에 유의한 차이가 있는지를 알아보고자 하였다. 평형을 유지하 는 데에는 하지 근골격계의 영향을 크게 받으며, 하지의 정렬뿐만 아 니라 근력에 의해서도 영향을 받으므로, 본 실험에서는 성별에 따른 근력의차이를 배제하기 위해 여성을 대상으로 실험하였다.

정적 안정성(static stability)은 신체의 움직임 없이 안정된 지지기저 면 내에 신체 중심을 위치시킴으로써 원하는 자세를 유지하는 능력 을 말하며, 동적 안정성(dynamic stability)은 신체를 움직이는 동안 안 정 또는 불안정한 지지기저면 내에서 신체 중심을 적절히 이동시킴 으로써 자세를 유지하는 능력을 말한다. ${ }^{20}$ Biodex Balance System은 이 러한 정적 및 동적 안정성을 측정하는 데에 높은 신뢰도를 보인 대표

Table 2. The differences in overall stability index (OSI), anterioposterior stability index (APSI), and mediolateral stability index (MLSI) of static and dynamic stability index among genu varum, valgum, and recurvatum group

\begin{tabular}{llcccc}
\hline & Control & Genu varum & Genu valgum & Genu recurvatum \\
\hline Static & OSI & $0.49 \pm 0.06$ & $0.52 \pm 0.09$ & $0.53 \pm 0.09$ & $0.54 \pm 0.07$ \\
& APSI & $0.34 \pm 0.07$ & $0.39 \pm 0.08$ & $0.40 \pm 0.09$ & $0.42 \pm 0.07$ \\
& $\mathrm{MLSl}^{+}$ & $0.30 \pm 0.07$ & $0.37 \pm 0.08$ & $0.37 \pm 0.07$ & $0.32 \pm 0.08$ \\
Dynamic & OSI & $1.15 \pm 0.14$ & $1.19 \pm 0.12$ & $1.17 \pm 0.10$ & $1.15 \pm 0.16$ \\
& $\mathrm{APSI}^{*}$ & $0.81 \pm 0.08$ & $0.84 \pm 0.09$ & $0.83 \pm 0.10$ & $0.91 \pm 0.18$ \\
& $\mathrm{MLSl}^{+}$ & $0.68 \pm 0.12$ & $0.79 \pm 0.08$ & $0.80 \pm 0.09$ & $0.70 \pm 0.16$ \\
\hline
\end{tabular}

Values are presented as mean \pm standard deviation. ${ }^{*} p<0.05 ;{ }^{*} p<0.01$.
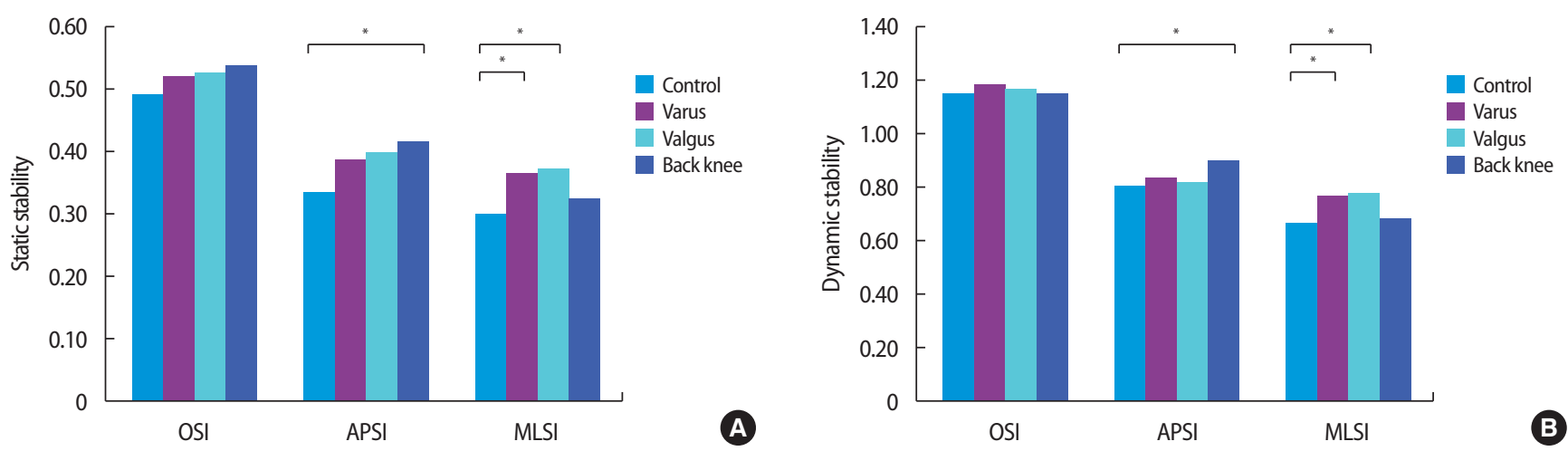

Figure 1. Comparison of overall stability index (OSI), anterioposterior stability index (APSI), and mediolateral stability index (MLSI) of static and dynamic stability index among genu varum, valgum, recurvatum and control group. 
적인 균형 측정 장비로, 이러한 정보를 통해 낙상 위험 지수 등을 평 가할수 있어 연구 목적으로나 임상에서도 많이 사용되고 있다.21-24

안굽이 무릎과 밖굽이 무릎은 전두면에서 나타나는 대표적인 정 렬 불량으로, 무릎관절의 내외측 안정성에 기여하는 능동적, 수동적 기전이 저하되어 있다. 능동적 기전은 근육에 의해 생성되는 힘으로 관절 안정성을 유지하는 능력을 말하며, 수동적 기전은 인대나 관절 주머니 등의 수동적 구조물에 의해 유지되는 능력을 말한다. 안굽이 무릎의 경우 가쪽 곁인대 및 관절 주머니가 신장되어 있고, 넙다리두 갈래근(biceps femoris)과 같은 무릎관절 가쪽 근육이 신장되어 있으 며, 안쪽 관절 간격이 소실되어 있다. 반면, 밖굽이 무릎의 경우 안쪽 곁인대 및 관절주머니가 신장되어 있고, 반힘줄모양근(semitendinosus)과 같은 안쪽 근육이 신장되어 있으며, 가쪽 관절 간격이 소실되 어 있다. ${ }^{17}$ 또한 하지 정렬의 변화로 체중지지선이 내, 외측으로 이동 함에 따라 체중지지의 비대칭을 증가시키고, 자세적 불안정성을 야 기시킨다. ${ }^{15}$ 본 연구에서 안굽이 무릎과 밖굽이 무릎이 정적 및 동적 인 상황하에서 모두 내외측 균형지수에 영향을 미친 것으로 나타났 으며, 이는 무릎관절의 내외측 안정성에 기여하는 이러한 기전들이 저하되어 있기 때문인 것으로 보인다. Chae 등 ${ }^{19}$ 은 안굽이 무릎이 정 적인 상태에서 내외측안정성에 영향을 미쳤으나, 동적인 상태에서는 내외측 안정성뿐만 아니라 전체균형지수, 전후균형지수에도 모두 영 향을 미친다고 하였다. 동적인 상태에서는 균형을 유지하는 데에 좀 더 도전적인 상황이 되므로, 안정성 유지에 더 많은 능력을 요구받게 되어 무릎 정렬 상태가 동적 안정성에 더 많은 영향을 미친 것이라고 하였다. 반면, Samaei 등 15 은 안굽이 무릎이 정적인 상태에서는 물론, 동적인 상태에서도 내외측 균형지수에만 유의한 영향을 미친다고 하 였으며, 밖굽이 무릎은 자세 안정성에 유의한 영향을 미치지 않는다 고 하였다. 본 연구에서는 안굽이 무릎과 밖굽이 무릎을 선별한 기준

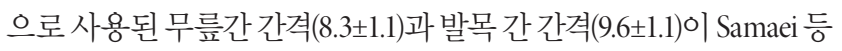
${ }^{15}$ 의 연구에서 측정된 값(무릎간 간격: 6.1 \pm 2.5 , 발목간 간격: $5.6 \pm 1.9$ )보 다 더 크므로, 안굽이 무릎과 밖굽이 무릎의 정도가 심하다고 볼 수 있으며, 따라서 안굽이 무릎뿐만 아니라 밖굽이 무릎에서도 내외측 균형지수에 유의한차이를 보였다고 할 수 있다.

젖힌 무릎은 무릎관절의 뒤쪽 관절주머니가 신장되어 있으며, 넙 다리네갈래근(quadriceps femoris)의 근력이 약화되어 있어 관절 안정 성이 저하되어 있다. 특히, 앞십자인대(anterior cruciate ligament)의 신 장으로 인해 임상적으로 손상의 위험이 증가되며, 노인에게서 낙상 의 위험을 증가시킨다. ${ }^{1,25-28}$ Siqueira 등 ${ }^{10}$ 은 젖힌 무릎이 바로 선 자세 에서의 $\mathrm{COP}$ 이동 속도를 증가시켜 안정성에 영향을 미친다고 하였 다. 눈을 감은 상태로 불안정한 면 위에서 측정한 $\mathrm{COP}$ 이동 속도는 가장 낮았는데, 그 이유로 무릎을 굽혀 자세를 유지하려는 운동 전략 을 실행시킴으로써 보상적 운동을 이끌었기 때문이라고 하였다. 본
연구 결과, 젖힌 무릎군이 정적 안정성과 동적 안정성에서 모두 전후 균형지수에 유의한 영향을 미친 것으로 나타났다. 본 연구에서 동적 안정성을 측정할 때 사용된 Biodex Balance System 발판의 불안정성 은 5 단계로, 선행 연구에서 불안정한 면을 제공하기 위해 사용한 Airex balance pad $\left(6 \mathrm{~cm}\right.$ thick, $57 \mathrm{~g} / \mathrm{dm}^{3}$ density)보다 정량적인 불안정성 을 제공할 수 있어 좀 더 객관적인 결과로 볼 수 있을 것이다. 향후 발 판의 불안정성이 더 높은 상태에서 실험이 진행될 경우 선행 연구의 결과와 비슷한 결과를 가질 수 있을 것이라 예상된다.

본 연구의 한계점은 대상자가 여성으로 한정되어 있어 성별의 차 이를 알 수 없었으며, 젖힌 무릎에 대한 상용화된 측정 방법이 없어 임의로 고안해낸 방법을 사용했다는 점이다. 향후 남성을 대상으로 추가적인 실험이 진행되어 성별의 차이가 안정성에 영향을 미치는지 알아볼 필요가 있을 것이다.

본 연구 결과, 무릎 정렬 상태가 자세 안정성에 영향을 미친 것으로 나타났으며, 이는 낙상이나 부상의 위험성을 높일 수 있음을 의미한 다. 특히, 안굽이 무릎과 밖굽이 무릎은 정적인 상태와 동적인 상태 하에서 모두 내외측 균형지수에 영향을 미친 것으로 나타났으며, 젖 힌 무릎은 전후균형지수에 영향을 미친 것으로 나타나, 무릎 정렬의 편차가 특정한 방향으로의 자세 안정성에 영향을 미친 것으로 나타 났다. 향후 이러한 정렬 이상을 치료하고 낙상이나 부상을 예방하기 위해 자세 안정성을 향상시키고자 하는 치료가 추가적으로 제공되 어야할 것이다.

\section{ACKNOWLEDGEMENTS}

The Research has been conducted by the Research Grant of Gwangju Health College in 2013 (No. 3013010).

\section{REFERENCES}

1. O’Brien K, Culham E, Pickles B. Balance and skeletal alignment in a group of elderly female fallers and nonfallers. J Gerontol A Biol Sci Med Sci. 1997;52(4):B221-6.

2. Lord SR, Clark RD, Webster IW. Postural stability and associated physiological factors in a population of aged persons. J Gerontol Med Sci. 1991; 46(3):M69-76.

3. Jang HJ, Kim SY, Kwon CS. Relationships between flexed posture, physical performance and psychosocial factors in elderly women. J Kor Phys Ther. 2014:26(5):358-64.

4. Park JW, Park S. Structural assessment of spastic hemiplegic foot using the foot posture index. J Kor Phys Ther. 2011;23(6):55-9.

5. Seo HJ, Kim JH, Son KK. The effect of different head positions with whole body vibration on muscle activation related to postural stability in standing. J Kor Phys Ther. 2014;26(3):156-62.

6. Park BJ, Kim JH. The effects of strengthening exercise of hip abductors 
on muscle strength and ambulation in patient with ACL reconstruction. J Kor Phys Ther. 2014;26(5):296-301.

7. Kantor E, Poupard L, Le Bozec S, et al. Does body stability depend on postural chain mobility or stability area? Neurosci Lett. 2001;308(2):12832.

8. Raymakers JA, Samson MM, Verhaar HJ. The assessment of body sway and the choice of the stability parameter(s). Gait Posture. 2005;21(1):4858.

9. Saha D, Gard S, Fatone S, et al. The effect of trunkflexed postures on balance and metabolic energy expenditure during standing. Spine. 2007;32 (15):1605-11.

10. Siqueira CM, Lahoz Moya GB, Caffaro RR, et al. Misalignment of the knees: Does it affect human stance stability. J Bodyw Mov Ther. 2011;15 (2):235-41.

11. Plisky PJ, Rauh MJ, Kaminski TW, et al. Star excursion balance test as a predictor of lower extremity injury in high school basketball players. J Orthop Sports Phys Ther. 2006;36(12):911-9.

12. Yang DJ, Park SK, Kang JI, et al. Effects of changes in postural alignment on foot pressure and balance of patients with stroke. J Kor Phys Ther. 2014;26(4): 226-33.

13. Johnson F, Leitl S, Waugh W. The distribution of load across the knee. A comparison of static and dynamic measurements. J Bone Joint Surg Br. 1980;62(3):346-9.

14. Tetsworth K, Paley D. Malalignment and degenerative arthropathy. Orthop Clin North Am. 1994;25(3):367-77.

15. Samaei A, Bakhtiary AH, Elham F, et al. Effects of genu varum deformity on postural stability. Int J Sports Med. 2012;33(6):469-73.

16. Anker LC, Weerdesteyn V, van Nes IJ, et al. The relation between postural stability and weight distribution in healthy subjects. Gait Posture. 2008;27(3):471-7.

17. Sharma L, Song J, Felson DT, et al. The role of knee alignment in disease progression and functional decline in knee osteoarthritis. JAMA.
2001;286(2):188-95.

18. Park S, Lee WJ, Park JW. Differences of onset timing between vastus medialis and lateralis during knee isometric contraction on individuals with genu varum or valgum. J Kor Phys Ther. 2014;26(1):9-14.

19. Chae YW, Park JW, Park S. The effect of postural stability on genu varum in young adults. J Kor Phys Ther. 2012;24(6):419-22.

20. Berger L, Klein C, Commandeur M. Evaluation of the immediate and midterm effects of mobilization in hot spa water on static and dynamic balance in elderly subjects. Ann Readapt Med Phys. 2008;51(2):84-95.

21. Aydog E, Bal A, Aydog ST, et al. Evaluation of dynamic postural balance using the biodex stability system in rheumatoid arthritis patients. Clin Rheumatol. 2006;25(4):462-7.

22. Cote KP, Brunet ME, Gansneder BM, et al. Effects of pronated and supinated foot postures on static and dynamic postural stability. J Athl Train. 2005;40(1):41-6.

23. Cachupe WJ, Shifflett B, Kahanov L, et al. Reliability of biodex balance system measures. Meas Phys Educ Exerc Sci. 2001;5:97-108.

24. Jeong HY, Choi JD. The effects of vestibular sensory stimulation training on balance and gait in the patients with stroke. J Kor Phys Ther. 2014; 26(5):365-71.

25. Cowan DN, Jones BH, Frykman PN, et al. Lower limb morphology and risk of overuse injury among male infantry trainees. Med Sci Sports Exerc. 1996;28(8):945-52.

26. Bonci CM. Assessment and evaluation of predisposing factors to anterior cruciate ligament injury. J Athl Train. 1999;34(2):155-64.

27. Devan MR, Pescatello LS, Faghri P, et al. A prospective study of overuse knee injuries among female athletes with muscle imbalances and structural abnormalities. J Athl Train. 2004;39(3):263-67.

28. Ramesh R, Von Arx O, Azzopardi T, et al. The risk of anterior cruciate ligament rupture with generalised joint laxity. J Bone Joint Surg Br. 2005; 87(6):800-3. 